\title{
Fat Adherence Syndrome
}

National Cancer Institute

\section{Source}

National Cancer Institute. Fat Adherence Syndrome. NCI Thesaurus. Code C118860.

Violation of Tenon's capsule with prolapse of orbital fat into the sub-Tenon's space. 